\title{
The plasma kallikrein-kinin system counterbalances the renin-angiotensin system
}

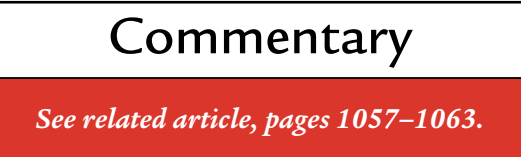

\begin{abstract}
Alvin H. Schmaier
University of Michigan, Departments of Internal Medicine and Pathology, 5301 Medical Science Research Building III, 1150 West Medical Center Drive, Ann Arbor, Michigan 48109-0640, USA.

Phone: (734) 647-3124; Fax: (734) 647-5669; E-mail: aschmaie@umich.edu.
\end{abstract}

J. Clin. Invest. 109:1007-1009 (2002). DOI:10.1172/JCI200215490.

The plasma kallikrein-kinin system (KKS), first recognized over 40 years ago, was originally believed to contribute to physiologic hemostasis. At the time, factor XII (Hageman factor, FXII) and the related proteins prekallikrein $(\mathrm{PK})$ and high molecular-weight kininogen $(\mathrm{HK})$ were known to be essential for efficient surface-activated blood coagulation, as measured in the activated partial thromboplastin time (APTT) test. Indeed, in this test, autoactivation of FXII in glass tubes promotes thrombin formation.

According to the then-current "contact activation" hypothesis, FXII activation on a negatively charged surface was thought to initiate hemostasis in a similar manner by a cascade of proteolytic reactions that culminate in thrombin formation. This model was undermined by the failure to identify such a physiologically relevant surface, coupled with evidence that individuals deficient in FXII, PK, or HK are free of bleeding disorders. In addition, the recognition that factor XI, whose deficiency is associated with bleeding, can be activated by thrombin provided a bypass mechanism that obviated a need for FXII to activate factor XI.

Only over the last 6 years have alternative explanations for the physiologic role of the KKS and its assembly and activation begun to emerge. Rather than assembling on a negatively charged surface such as that used in the APTT test, the proteins of the plasma KKS are now known to bind a multiprotein receptor complex in the intravascular compartment. As shown in Figure 1, HK, the critical regulator of plasma KKS assembly and activation, binds an endothelial cell surface receptor complex containing cytokeratin 1 (CK1), urokinase plasminogen activator receptor ( $\mathrm{uPAR}$ ), and gC1qR (1-6). Recent studies with cultured human umbilical vein endothelial cells indicate that FXII can also bind to this receptor (7), but that this interaction is highly regulated. Plasma concentrations of HK completely block FXII binding to the multiprotein receptor complex. Further, FXII binding requires a 30-fold higher free $\mathrm{Zn}^{2+}$ concentration than does HK, which can only be

\section{Figure 1}

Assembly and activation of the plasma KKS on endothelial cells. Plasma PK circulates in complex with HK. The $\mathrm{HK} \bullet \mathrm{PK}$ complex binds to a multiprotein receptor complex that consists of cytokeratin 1 (CK1), urokinase plasminogen activator receptor ( $\mathrm{UPAR}$ ) and gC1qR. The proteins of the HK•PK receptor complex co-localize on endothelial cell membranes. When $\mathrm{HK} \bullet P K$ binds to endothelial cells, PK is rapidly converted to kallikrein (K) by the enzyme prolylcarboxypeptidase (PRCP), which is constitutively active on endothelial cell membranes. The resulting kallikrein autodigests its receptor, HK, to liberate bradykinin (BK), which can liberate tissue plasminogen activator (tPA), nitric oxide (NO), and prostacyclin $\left(\mathrm{PGl}_{2}\right)$ from endothelial cells. Kallikrein also activates FXII, which binds to the same multiprotein receptor complex as HK in its absence. In this revised hypothesis for assembly and activation of the proteins of the plasma KKS, FXII is activated by kallikrein after PK activation. ScuPA, single chain urokinase plasminogen activator.

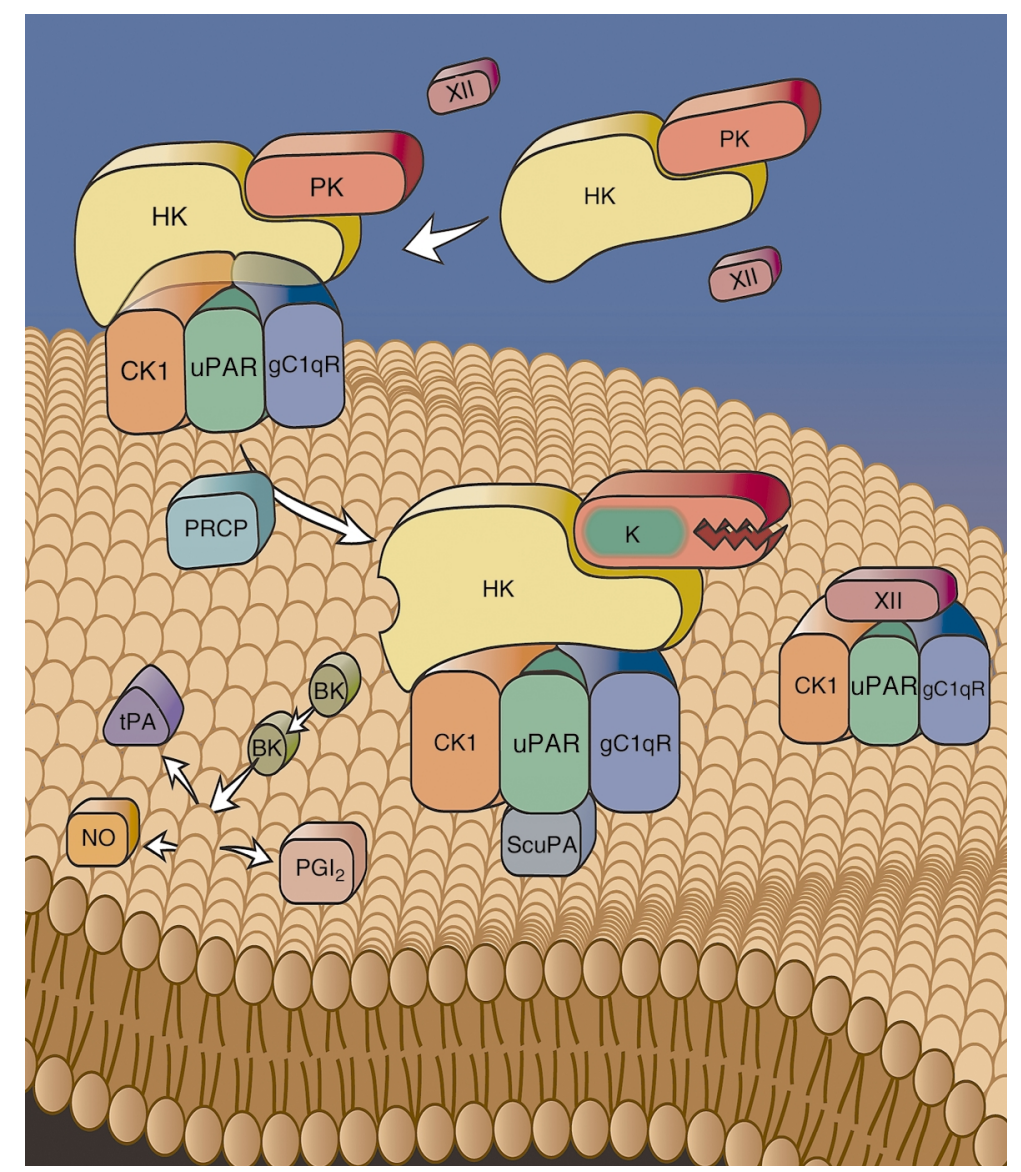




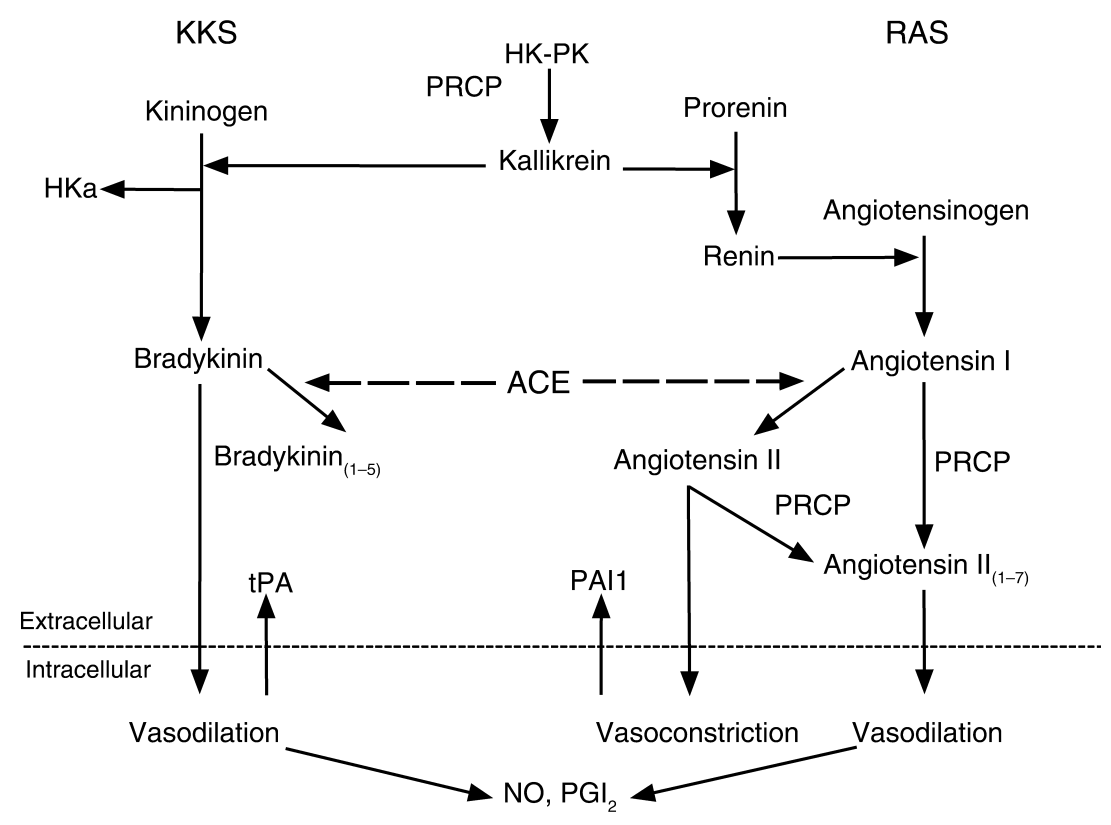

Figure 2

The interaction between the plasma KKS and RAS. Plasma kallikrein converts prorenin to renin, and renin has the ability to convert angiotensinogen to angiotensin I. Angiotensin-converting enzyme (ACE) converts inactive angiotensin I to the vasoconstrictor angiotensin II. Angiotensin II stimulates plasminogen activator inhibitor 1 (PAI1) release from endothelial cells. At the same time ACE degrades bradykinin into bradykinin ${ }_{(1-7)}$ (not shown) or bradykinin $(1-5)$, a peptide with thrombin inhibitory activity. PRCP is the enzyme that degrades angiotensin II or angiotensin I to the vasodilating peptide, angiotensin $\mathrm{II}_{(1-7)}$. Angiotensin $\mathrm{II}_{(1-7)}$ stimulates $\mathrm{NO}$ and $\mathrm{PGI}_{2}$ formation, which potentiates the effects of bradykinin. PRCP also has the ability to convert PK to kallikrein. Formed kallikrein digests kininogens to liberate bradykinin, leaving a kinin-free kininogen ( $\mathrm{HKa}$ ) that has anti-proliferative and anti-angiogenic properties. Thus, PRCP, the same enzyme that degrades the vasoconstrictor angiotensin II, leads to the increased formation of the vasodilators bradykinin and angiotensin $\mathrm{II}_{(1-7)}$. Finally, the resulting bradykinin stimulates $\mathrm{tPA}, \mathrm{NO}$, and $\mathrm{PGI}_{2}$ formation, thus counterbalancing the prothrombotic effect of angiotensin II.

achieved in a milieu of activating platelets or other cells (7). Thus, under physiologic conditions, HK binds to this endothelial cell complex but FXII is prevented from doing so.

\section{Mechanisms of KKS activation}

This interaction of HK with its endothelial cell-surface receptor is key to the regulated activation of $\mathrm{PK}$, which circulates in the plasma in a complex with HK. Work with cultured endothelial cells suggests a novel mechanism by which HK-bound PK is rapidly converted to kallikrein in a process that is independent of FXII $(8,9)$. On cultured endothelial cells and cell matrices, FXII activation occurs subsequent to PK activation $(9,10)$. Activated forms of FXII thus do not initiate PK activation, although they can feed back to increase the rate and extent of its activation. Activation of PK - unlike that of a structurally related zymogen, factor XI - can therefore proceed even in the absence of factor XIIa (11). As shown in
Figure 1, endothelial cell-associated active kallikrein then cleaves HK to liberate bradykinin. The assembly of the $\mathrm{HK} \bullet \mathrm{PK}$ complex on endothelial cells is thus predicted to lead to constitutive production of bradykinin, which can then activate the bradykinin $B_{2}$ receptor. Activation of this receptor regulates vascular tone by stimulating NO formation in endothelial cells (12).

This mechanism for bradykinin production depends on the activity of an endothelial cell-borne PK activator, whose identity was unknown until quite recently. We have now reported that the serine protease prolylcarboxypeptidase (PRCP, lysosomal carboxypeptidase, angiotensinase $\mathrm{C}$ ) represents one such enzyme (13). Its $K_{\mathrm{m}}$ for $\mathrm{PK}(7 \mathrm{nM})$ is consistent with a physiologic role as a specific PK activator (13), and it is found on endothelial cell membranes and in the endothelial cell-endomembrane system that communicates between the cell's lysosomal and membrane compartments. Previous investigations by Erdös and colleagues identified angiotensin II $\left(K_{m}=0.2 \mathrm{mM}\right)$ and bradykinin $\left(K_{m}=1\right.$ $\mathrm{mM})$ as substrates of the same processing enzyme (14). PRCP can activate the biologically inert angiotensin I or the vasoconstrictor angiotensin II to form angiotensin $\mathrm{II}_{1-7}$, a biologically active peptide that induces vasodilation by stimulating NO formation $(15,16)$ (Figure 2). The finding that PRCP also activates $\mathrm{PK}$ indicates that it can produce two biologically active peptides, bradykinin and angiotensin $\mathrm{II}_{1-7}$, each of which can reduce blood pressure, counterbalancing the vasoconstrictive effects of angiotensin II.

\section{The KKS in thrombosis}

The known ability of angiotensin II to induce plasminogen activator inhibitor 1 secretion implicates the reninangiotensin system (RAS) in promoting thrombosis (17). The recent findings that PRCP activates PK (13) and inactivates angiotensin II (14) indicate an important and previously unappreciated interaction between the plasma KKS and the RAS (Figure 2), suggesting that these pathways jointly not only regulate blood pressure, but may also influence thrombosis. Two other known interactions between the KKS and the RAS have already been documented: plasma kallikrein can activate prorenin to renin (18), while angiotensin-converting enzyme (kininase II) can convert bradykinin into the thrombin-inhibitory peptide bradykinin ${ }_{1-5}$ and can also convert angiotensin I to angiotensin II (19, 20). The plasma KKS is therefore predicted to be anticoagulant and profibrinolytic $(8,16)$. Indeed, bradykinin is a potent stimulator of $\mathrm{NO}$ formation, prostacyclin liberation, and tissue plasminogen activator release as well as an inhibitor of thrombin $(12,20-22)$. Moreover, kallikrein is a kinetically favorable activator of single-chain urokinase (8). Considering the established role of bradykinin as a hypotensive peptide, it appears that the plasma KKS serves as a physiologic counterbalance to the hypertensive, prothrombotic RAS (Figure 2).

\section{Evidence for the physiologic activities of the KKS}

To date there has been a paucity of animal models in which the role of the plasma KKS can be studied. One such model is the bradykinin $\mathrm{B}_{2}$ receptor knockout mouse $\left(B K B_{2} R^{-/-}\right)$. Mice with 
this defect have cardiac hypertrophy, chamber dilatation, and elevated left ventricular end-diastolic pressure, and they show exaggerated vasopressor responses to angiotensin II (23). In the presence of angiotensin II infusion, these animals have increased blood pressure and reduced renal blood flow (24). They also experience a diminished cardioprotective benefit from angiotensin I receptor antagonists and angiotensin-converting enzyme inhibitors, relative to the response of wild-type mice after ischemia-induced heart failure (25). This animal model links bradykinin with angiotensin II in the development of cardiac disease, but leaves open the question of their interrelationship in the area of thrombosis, where the expected phenotype is by no means obvious. While it is possible that the $B K B_{2} R^{-/-}$mouse will prove to be prothrombotic due to impaired tPA, NO, and prostacyclin liberation, the animal could equally be protected from thrombosis as a result of reduced metabolism of bradykinin.

In this issue of the JCI, Han et al. present another animal model that addresses a different prediction of the model proposed above, namely the idea that bradykinin is continuously formed in the intravascular compartment. These authors have studied mice deficient in the $\mathrm{C} 1$ inhibitor (C1 INH) (26), an inborn error that, in humans, is associated with hereditary angioedema (HAE). C1 INH, a member of the serpin family of serine proteinase inhibitors, is a major inhibitor of $\mathrm{C} 1 \mathrm{r}$, C1s, plasma kallikrein, and factor XIIa. $\mathrm{C} \mathrm{INH}^{-/-}$mice show reduced plasma C4 levels and low plasma total complement levels from chronic complement activation as a result of their $\mathrm{C} 1 \mathrm{INH}$ deficiency. It is of interest that these mice survive gestation, since homozygous humans deficient in $\mathrm{C} 1 \mathrm{INH}$ have never been described. Both homozygous and heterozygous mutant mice exhibit increased vascular permeability after injection of Evans blue dye. This phenotype can be corrected by providing exogenous $\mathrm{C} 1 \mathrm{INH}$.

Crucially, mice doubly deficient in both $\mathrm{C} 1 \mathrm{INH}$ and the bradykinin $\mathrm{B}_{2}$ receptor are protected from this effect, indicating that the increase in vascular permeability is mediated by bradykinin (22). These data indicate that bradykinin is the key mediator of the edema in these animals, and presumably in
HAE patients as well. Since these animals show constitutively increased permeability, due to liberated bradykinin, plasma kallikrein formation also occurs continuously in the intravascular compartment. This interpretation is consistent with the mechanism of PK activation shown in Figure 1. The $\mathrm{C} 1 \mathrm{INH}^{-/}$ mice provide in vivo support for this model of intravascular kallikrein formation, which was developed based on cell culture studies. There is presently no evidence that $\mathrm{C} 1 \mathrm{INH}$ inhibits endothelial surface-bound kallikrein as it does in solution, but it will be important to test this possibility.

In sum, recent investigations indicate a physiologic basis for assembly and activation of the plasma KKS. These mechanisms for assembly and activation of the plasma KKS suggest that it is the physiologic counterbalance to the RAS. The interaction of these two systems represents a direct link between blood pressure regulation and thrombosis. The details of this emerging paradigm are likely to be further elucidated by additional animal models such as the one described here by Han et al. (26).

\section{Acknowledgments}

I appreciate the critical review of this Commentary by Lilli Petruzzelli and Zia Shariat-Madar. This work is supported by NIH grants HL52779, HL57346, and HL65194.

1. Hasan, A.A.K., Zisman, T., and Schmaier, A.H. 1998. Identification of cytokeratin 1 as a binding protein and presentation receptor for kininogen on endothelial cells. Proc. Natl. Acad. Sci. USA 95:3615-3620

2. Shariat-Madar, Z., Mahdi, F., and Schmaier, A.H 1999. Mapping the cell binding site on cytokeratin 1. J. Biol. Chem. 274:7137-7145.

3. Colman, R.W., et al. 1997. Binding of high molecular weight kininogen to human endothelial cells is mediated via a site within domains 2 and 3 of the urokinase receptor. J. Clin. Invest. 100:1481-1487.

4. Herwald, H., Dedio, J., Kellner, R., Loos, M., and Muller-Esterl, W. 1996. Isolation and characterization of the kininogen-binding protein $\mathrm{p} 33$ from endothelial cells. J. Biol. Chem. 271:13040-13047.

5. Joseph, K., Ghebrehiwet, B., Peerschke, E.I.B., Reid, K.B.M., and Kaplan, A.P. 1996. Identification of the zinc-dependent endothelial cell binding protein for high molecular weight kininogen and factor XII: identity with the receptor that binds to the globular "heads" of C1q (gC1q-R). Proc. Natl. Acad. Sci. USA. 93:8552-8557.

6. Mahdi, F., Shariat-Madar, Z., Todd, R.F., III Figueroa, C.D., and Schmaier, A.H. 2001. Expression and co-localization of cytokeratin 1 and urokinase plasminogen activator receptor on endothelial cells. Blood. 97:2342-2350.

7. Mahdi, F., Shariat-Madar, Z., Figueroa, C.D., and Schmaier, A.H. 2002. Factor XII interacts with the multiprotein assembly of urokinase plasminogen activator receptor, gC1qR, and cytokeratin 1 on endothelial cell membranes. Blood. In press.
8. Motta, G., Rojkjaer, R., Hasan, A.A.K., Cines, D.B., and Schmaier, A.H. 1998. High molecular weight kininogen regulates prekallikrein assembly and activation on endothelial cells. A novel mechanism for contact activation. Blood. 91:516-528.

9. Rojkjaer, R., Hasan, A.A.K., Motta, G., Schousboe, I., and Schmaier, A.H. 1998. Factor XII does not initiate prekallikrein activation on endothelial cells. Thromb. Haemost. 80:74-81.

10. Motta, G., Shariat-Madar, Z., Mahdi, F., Sampaio, C.A.M., and Schmaier, A.H. 2001. Assembly and activation of high molecular weight kininogen and prekallikrein on cell matrix. Thromb. Haemost. 86:840-847.

11. Shariat-Madar, Z., Mahdi, F., and Schmaier, A.H. 2001. Factor XI assembly and activation on human umbilical vein endothelial cells in culture. Thromb. Haemost. 85:544-551.

12. Zhao, Y., et al. 2001. Assembly and activation of the $\mathrm{HK} \bullet \mathrm{PK}$ complex on endothelial cells results in bradykinin liberation and NO formation. Am. Physiol. Heart Circ. Physiol. 280:H1821-H1829.

13. Shariat-Madar, Z., Mahdi, F., and Schmaier, A.H 2002. Identification and characterization of prolyl carboxypeptidase as an endothelial cell prekallikrein activator. J. Biol. Chem. In press.

14. Odya, C.E., Marinkovic, D.V., Hammon, K.J., Stewart, T.A., and Erdös, E.G. 1978. Purification and properties of prolylcarboxypeptidase (angiotensinase C) from human kidney. J. Biol. Chem. 253:5927-5931.

15. Santos, R.A.S., Brosnihan, K.B., Jacobsen, D.W., DiCorleto, P.E., and Ferrario, C.M. 1992. Produc tion of angiotensin-(1-7) by human vascular endothelium. Hypertension. 19(Suppl. 2):II56-II61.

16. Ren, Y.L., Garvin, J.L., and Carretero, O.A. 2002 Vasodilator action of angiotensin-(1-7) on isolated rabbit afferent arterioles. Hypertension. 39:799-802.

17. Brown, N.J., and Vaughan, D.E. 2000. Prothrombotic effects of angiotensin. Adv. Int. Med. 45:419-429.

18. Sealey, J.E., Atlas, S.A., and Laragh, J.H. 1978. Linking the kallikrein and renin systems via activation of inactive renin. New data and a hypothesis. Am. J. Med. 65:994-1000.

19. Yang, H.Y.T., Erdös, E.G., and Levin, Y. 1971. A dipeptide carboxypeptidase that converts angiotensin I and inactivates bradykinin. Biochim. Bipophys. Acta. 214:374-376.

20. Hasan, A.A.K., Amenta, S., and Schmaier, A.H 1996. Bradykinin and its metabolite, ARG-PRO PRO-GLY-PHE, are selective inhibitors of $\alpha$-thrombin-induced platelet aggregation. Circulation. 94:517-528

21. Hong, S.L. 1980. Effect of bradykinin and thrombin on prostacyclin synthesis in endothelial cells from calf and pig aorta and human umbilical cord vein. Thromb. Res. 18:787-795.

22. Brown, N.J., Nadeau, J.H., and Vaughan, D.E. 1997. Selective stimulation of tissue-type plasminogen activator (tPA) in vivo by infusion of bradykinin. Thromb. Haemost. 77:522-525.

23. Madeddu, P., et al. 1997. Cardiovascular phenotype of a mouse strain with disruption of bradykinin $\mathrm{B}_{2}$-receptor gene. Circulation. 96:3570-3578

24. Cervenka, L., et al. 2001. Angiotensin II-induced hypertension in bradykinin $\mathrm{B}_{2}$ receptor knockout mice. Hypertension. 37:967-973.

25. Yang, X.-P., et al. 2001. Diminished cardioprotective response to inhibition of angiotensin-converting enzyme and angiotensin II type 1 receptor in $\mathrm{B}_{2}$ kinin receptor gene knockout mice. Circ. Res. 88:1072-1079

26. Han, E.D., MacFarlane, R.C., Mulligan, A.N., Scafidi, J., and Davis, A.E., III. 2002. Increased vascular permeability in C1 inhibitor-deficient mice mediated by the bradykinin type 2 receptor. J. Clin. Invest. 109:1057-1063. DOI:10.1172/JCI200214211. 